\title{
Changes over time in inflammatory and structural lesions at the sacroiliac joint in children with spondyloarthritis exposed and unexposed to tumor necrosis factor inhibitor
}

Timothy G. Brandon', Rui Xiao², Rosemary G. Peterson ${ }^{3}$, Nancy A. Chauvin ${ }^{4}$, Michael L. Francavilla ${ }^{5}$, David M. Biko ${ }^{5}$, Dax G. Rumsey ${ }^{6}$, Matthew L. Stoll ${ }^{7}$ and Pamela F. Weiss ${ }^{8^{*}}$ (D)

\begin{abstract}
Background: The objective of this work was to describe magnetic resonance imaging (MRI) changes over time in inflammatory and structural lesions at the sacroiliac joint (SIJ) in children with spondyloarthritis (SpA) exposed and unexposed to tumor necrosis factor inhibitor (TNFi).

Methods: This was a retrospective, multicenter study of SpA patients with suspected or confirmed sacroiliitis who underwent at $\geq 2$ pelvic MRI scans. Images were reviewed independently by 3 radiologists and scored for inflammatory and structural changes using the Spondyloarthritis Research Consortium of Canada (SPARCC) SIJ inflammation score (SIS) and structural score (SSS). Longitudinal, quantitative changes in patient MRI scans were measured using descriptive statistics and stratified by TNFi exposure. We used an average treatment effects (ATE) regression model to explore the average effect of TNFi exposure over time on inflammatory and structural lesions, adjusting for baseline lesion scores.

Results: Forty-six subjects were evaluated using the SIS $(n=45)$ and SSS $(n=18)$. Median age at baseline imaging was 13.6 years, $63 \%$ were male and $71 \%$ were white. Twenty-three subjects (50\%) were TNFi exposed between MRI studies. The median change in SIS in TNFi exposed and unexposed subjects with a baseline SIS $\geq 0$ was -20.7 and - 14.3, respectively $(p=0.09)$. Eleven (85\%) TNFi exposed and $8(89 \%)$ unexposed subjects with a baseline SIS $\geq 0$ met the SIS minimal clinically important difference (MCID; $\geq 2.5)$. Using the ATE model adjusted for baseline SIS, the average effect of TNFi on SIS in patients with a baseline SIS $\geq 2$ was $-14.5(p<0.01)$. Unadjusted erosion change score was significantly worse in TNFi unexposed versus exposed subjects $(p=0.03)$ but in the ATE model the effect of TNFi was not significant.
\end{abstract}

\footnotetext{
* Correspondence: weisspa@chop.edu

${ }^{8}$ Department of Pediatrics, Division of Rheumatology and Center for Pediatric Clinical Effectiveness at the Children's Hospital of Philadelphia and Center for Clinical Epidemiology and Biostatistics, Perelman School of Medicine at the University of Pennsylvania, 2716 South Street, Room 11121, Philadelphia, PA 19104, USA

Full list of author information is available at the end of the article
}

(c) The Author(s). 2021 Open Access This article is licensed under a Creative Commons Attribution 4.0 International License, which permits use, sharing, adaptation, distribution and reproduction in any medium or format, as long as you give appropriate credit to the original author(s) and the source, provide a link to the Creative Commons licence, and indicate if changes were made. The images or other third party material in this article are included in the article's Creative Commons licence, unless indicated otherwise in a credit line to the material. If material is not included in the article's Creative Commons licence and your intended use is not permitted by statutory regulation or exceeds the permitted use, you will need to obtain permission directly from the copyright holder. To view a copy of this licence, visit http://creativecommons.org/licenses/by/4.0/ The Creative Commons Public Domain Dedication waiver (http://creativecommons.org/publicdomain/zero/1.0/) applies to the data made available in this article, unless otherwise stated in a credit line to the data. 
Conclusion: This study quantitatively describes how lesions in the SIJS on MRI change over time in patients exposed to TNFi versus unexposed. Follow-up imaging in TNFi exposed patients showed greater improvement than the unexposed group by most metrics, some of which reached statistical significance. Surprisingly, a majority of TNFi unexposed children with a baseline SIS $\geq 2$ met the SIS MCID. Additional studies assessing the short and longterm effects of TNFi on inflammatory and structural changes in juvenile SpA are needed.

Keywords: SACROILIAC JOINT, MAGNETIC RESONANCE IMAGING, PEDIATRICS, SPONDYOLARTHRITIS, DISEASE PROGRESSION, TUMOR NECROSIS FACTOR INHIBITORS

\section{Background}

Juvenile spondyloarthritis (SpA) encompasses a group of heterogeneous conditions characterized by chronic inflammatory arthritis, enthesitis, inflammatory bowel disease, acute anterior uveitis, psoriasis, and HLA-B27 positivity. Up to 30 $\%$ of children with $\mathrm{SpA}$ develop sacroiliitis within several years of diagnosis [1]. If untreated, this can progress to ankylosing spondylitis (AS), an inflammatory disease that causes joint fusion and leads to permanent functional impairment [1-4] . Compared to adults with AS, children with SpA have worse functional outcomes, including sacroiliac radiographic scores and spinal deformity $[5,6]$. While similarities exist between juvenile and adult SpA, there are distinct phenotypic differences that warrant specific focus on juvenile disease [711]. In comparison to adult-onset disease, juvenile-onset disease is associated with a higher prevalence of affected females [9], more peripheral disease [8, 11], less back pain [10], and less involvement of the spine $[9,11]$. Additionally, several studies demonstrate that adult patients with juvenile-onset disease have more radiographic changes of the hips and significantly higher risk of hip replacement [7-9], making early recognition critical to preserve long-term functional outcomes.

In adults, TNF-inhibitor (TNFi) medications have been shown to help symptomatically and appear to lead to faster resolution of the inflammatory phase and start of tissue repair at sites of erosion [12-14]. It is hypothesized that TNFi medications may slow the progression toward new bone formation and ankylosis, although this has not yet been definitively proven. In children, the effectiveness of TNFi medications for axial disease and magnitude of response on MR imaging of the sacroiliac joints (SIJs) is not known. In a study by Bray et al of adolescents and young adults with sacroiliitis, SPARCC inflammation scores improved after TNFi exposure while structural scores did not; however, there was not a TNFi naïve comparison group [15]. Further characterization of the changes over time in in inflammatory and structural lesions at the SIJ in children with SpA exposed and unexposed to TNFi is needed.

MRI has become increasingly preferred as the primary imaging modality for evaluating disease in the sacroiliac joint due to its ability to detect inflammation prior to development of bony damage detectable by radiographs. Additionally, in children, radiographs have been shown to have high rates of misclassification of sacroiliitis using MRI as the reference standard with false positive radiographs occurring more frequently than false negative radiographs [16]. The Spondyloarthritis Research Consortium of Canada (SPARCC) sacroiliac inflammation (SIS) and structural scores (SSS) are validated measures to assess the progression of sacroiliitis on MRI and have been found to be both reliable and responsive to therapeutic intervention in adults [17-19]. Both have also shown to be reliable and feasible measures in children with suspected or confirmed juvenile SpA [20].

Characterization of the effect of biologic agents on the progression of sacroiliitis in children with SpA is critical to understanding the underlying disease pathophysiology and choosing the most effective treatments. The rarity of juvenile SpA limits the feasibility of randomized clinical trials and necessitates performing innovative observational studies. We aimed to evaluate the change in the SPARCC SIS and SSS lesions over time in TNFi-exposed versus TNFi-unexposed patients.

\section{Methods}

This study's protocol was reviewed and approved by the University of Alabama at Birmingham's (IRB-160701011) and Children's Hospital of Philadelphia's (IRB 16-013015) Committees for the Protection of Human Subjects and the ethics committee at the University of Alberta (REB Pro00081651). Waivers of consent and HIPAA authorization were granted as procedures represented minimal risk to the subjects and did not adversely affect the rights and welfare of the subjects.

\section{Study population}

This was a retrospective cohort study of children with SpA and suspected sacroiliitis who underwent at least two pelvic MRI scans spaced at least 12 weeks or 2 years apart for assessment of the SIS and SSS change scores, respectively, between January 2005 and July 2020. Studies were obtained at the treating physician's discretion. Eligible cases were aged 0-19 years old at time of baseline imaging. Patients with $\geq 4$ weeks of TNFi exposure prior to the first MRI were excluded. Pelvic MRI studies were performed at the Children's Hospital of Philadelphia (Philadelphia, Pennsylvania, USA), Children's of 
Alabama (Birmingham, Alabama, USA), Stollery Children's Hospital (Edmonton, Alberta, Canada), and the Grey Nuns' Hospital (Edmonton, Alberta, Canada). Since this was not a prospective protocolized study, imaging sequences were slightly different at the three participating institutions, but all studies were performed on high field-strength magnets $(1.5 \mathrm{~T}$ or $3.0 \mathrm{~T})$. As long as there was a semicoronal T1-weighted (T1W) and short tau inversion recovery (STIR) or other equivalent fluidsensitive sequence of the sacroiliac joints, subtle differences in MRI protocols did not preclude SPARCC SIS or SSS scoring. Imaging studies that did not include either the fluid-sensitive or T1W coronal oblique sequence were excluded for evaluation of the SIS and SSS, respectively. Radiology archiving systems and clinic records were queried to identify potentially eligible subjects and abstract physical examination reports, disease activity measures, patient-reported pain and global assessment scales, and medication use in the period leading up to and between the MRIs.

\section{MRI evaluation}

All cases were centrally collected, anonymized, and scored independently in pairs blinded to time point. Three radiologists (NC, MF, DB) with extensive experience in sacroiliac joint imaging used the online viewing and scoring system at www.CaREArthritis.com to score the Digital Imaging and Communication in Medicine (DICOM)-based cases. The radiologists scored the SPARCC SIS, SSS, or both depending on what MRI sequences were available. For patients with more than two studies, the first and second study were included in the analysis for SIS to provide the best estimate of rate of change in acute inflammation, while the first and last qualifying studies were included in the analysis for SSS to provide a sufficient time window for structural changes to accrue. Patients with an SIS of zero on both MRI scans were included because, clinically, axial arthritis was suspected and excluding those patients could disproportionately bias the results for the TNFi unexposed group away from a null finding.

The SIS divides each sacroiliac joint into quadrants and scores presence, depth, and intensity of bone marrow edema (BME) on STIR MRI sequences and has been demonstrated to be reliable and valid in the pediatric population for both status and change scores [20-22]. Six consecutive semicoronal slices through the cartilaginous portion of the joint are scored for BME (total score 0-72). The accepted minimal clinically important difference (MCID) in the SPARCC SIJ inflammation score is 2.5, established during a randomized, placebo-controlled clinical trial of adalimumab where participants reported their global evaluation of change on a scale of "much worse" to "much better" at each MRI visit [17]. A SPARCC SIS score of $\geq 2$ was used as a surrogate for meeting the Assessment of Spondyloarthritis International Society (ASAS) definition of a positive MRI [23].

The SSS assesses a spectrum of structural lesions of the SIJ on MRI including erosion, backfill, fat metaplasia, and ankylosis on 5 consecutive slices through the cartilaginous part of the joint and has been shown to be reliable for both status and change scores [20, 22]. These components are scored 0-20 (backfill and ankylosis) or $0-40$ (erosion and fat metaplasia). For the evaluation of pediatric cases, sclerosis is also included and scored 0-40 [20]. Standards of interpretation and terminology were established by having the radiologists view training modules and conduct calibration exercises using 30 reference cases as publicly available on www.CaREarthritis.org. All raters previously completed calibration exercises for both the SPARCC SIS and SSS, achieving acceptable reliability. For cases on which the 2 raters disagreed about the presence/absence of SIS or SSS components, the case was scored by a 3rd rater. Scores for the SIS and all SSS components were averaged across the two raters who agreed about the presence or absence of a lesion to provide a final score for each case.

\section{Analysis}

Study cohort demographics, clinical features, and component scores assigned by radiologists were summarized using standard descriptive statistics. Intraclass correlation coefficients (ICCs) were calculated to assess interrater reliability using two-way mixed-effects models measuring absolute agreement in scoring by radiologists. Interpretation of ICCs were as follows: ICC $<0.40$ was poor, $0.40 \leq$ ICC $<$ 0.75 was fair to good, and ICC $\geq 0.75$ was excellent [24]. These interpretation thresholds were used in the evaluation of the SPARCC SIS and SSS status and change scores in pediatrics [20-22]. Spearman's correlation was used to assess the relationship of change in pain score with change in SIS.

We used regression adjustment in an average treatment effects (ATE) model, allowing for a comparison of the sample mean difference between those treated and those untreated, to explore the average effect of TNFi exposure between scans on inflammatory and structural lesions, adjusting for baseline lesion scores. Subjects were considered TNFi exposed if they were treated with a TNFi for 90 days or more between the two MRI studies. To test for robustness of our choice of exposure window, sensitivity analyses were performed with different windows of TNFi treatment prior to the second MRI $(60,90,120$, and 180 days). Statistical analyses were conducted using Stata 14.2 (StataCorp. 2015, Stata Statistical Software: Release 14. College Station, TX: StataCorp LP). 


\section{Results}

\section{Subjects}

A total of 57 unique patients from three tertiary care centers were available for evaluation. After exclusion of 11 patients due to TNFi exposure $\geq 4$ weeks prior to baseline imaging, 46 eligible patients were included in the analysis. Demographic and clinical characteristics of the patients at the time of baseline imaging are shown in Table 1 . The majority of patients in this cohort were diagnosed with enthesitis-related arthritis (89\%). The remaining patients were diagnosed with either psoriatic arthritis, undifferentiated arthritis, or inflammatory bowel disease-associated arthritis. SIS was assessed in 45 cases and SSS was assessed in 18 cases. The median age at the time of baseline imaging was 13.6 years (IQR: 11.4-15.4), 63\% were male and $71 \%$ were white. The median duration of disease at the time of baseline imaging was 11.3 months (IQR: 3.7-23.7) and time between imaging studies included for assessment of change in the SIS and SSS were 14.5 (IQR: 6.0-25.3) and 46.1 (IQR: 27.4-52.7) months, respectively. Twenty-three subjects (50\%) were TNFi-exposed between the two MRI scans. Four (9\%), 14 (30\%) and 14 (30\%) received infliximab, etanercept or adalimumab respectively; six subjects received more than one TNFi sequentially between SISeligible MRI studies and six subjects received more than one TNFi between SSS-eligible MRI studies. One subject was considered TNFi unexposed by study exposure definition but received 67 days of TNFi between MRI studies. There were four patients exposed to a non-TNFi biologic disease-modifying antirheumatic drug (bDMARD); all were also TNFi exposed. Three of four started the TNFi first and, of those, two did not start the non-TNFi until after the change in inflammation follow-up assessment period (postMRI 2). One of the four patients only had sequences available to evaluate the SIS.

\section{Interrater reliability}

The interrater reliability across the three radiologists was excellent for the SIS status scores $(\mathrm{ICC}=0.89)$ and fair to good for the SSS components' status scores for erosion ( ICC $=0.57$ ), fat metaplasia ( $\mathrm{CCC}=0.52$ ), and ankylosis $(\mathrm{ICC}=0.72)$. Agreement was poor for the remaining SSS components: sclerosis $(\mathrm{ICC}=0.32)$ and backfill (ICC $=0.33)$. The relatively low reliability for some of the SSS components was partially due to the low prevalence of reported lesions in this pediatric cohort with only $13(36.1 \%)$ erosion, 6 (23.1\%) sclerosis, 2 (5.6\%) fat metaplasia, 3 (8.3\%) backfill, and 2 (5.6\%) ankylosis cases.

\section{SPARCC inflammation scores}

Forty-five subjects (98\%) had 2 evaluable SIS studies at least 12 weeks apart. Twenty-three (51\%) and 22 (49\%) subjects had an SIS of $<2$ and $\geq 2$ on the baseline scan, respectively. Cases with an SIS $\geq 2$ versus

Table 1 Subject characteristics

\begin{tabular}{|c|c|c|c|}
\hline & $\begin{array}{l}\text { All subjects } \\
(N=46)\end{array}$ & $\begin{array}{l}\text { TNFi exposed } \\
(N=23)\end{array}$ & $\begin{array}{l}\text { TNFi unexposed } \\
(N=23)\end{array}$ \\
\hline Age, years (Median, IQR) & $13.6(11.4,15.4)$ & $13.1(11.4,15.3)$ & $13.9(11.4,15.4)$ \\
\hline Sex, male & $29(63.04 \%)$ & $13(56.52 \%)$ & $16(69.57 \%)$ \\
\hline Race, white & $30(71.43 \%)[N=42]$ & $16(72.73 \%)[N=22]$ & $14(70.00 \%)[N=20]$ \\
\hline HLA-B27 positive & $23(50.00 \%)$ & $14(60.87 \%)$ & $9(39.13 \%)$ \\
\hline Hip arthritis & $12(26.09 \%)$ & $8(34.78 \%)$ & $4(17.39 \%)$ \\
\hline Lower back pain & $27(58.70 \%)$ & $12(52.17 \%)$ & $15(65.22 \%)$ \\
\hline Morning stiffness (> $15 \mathrm{~min}$ ) & $20(45.45 \%)[N=44]$ & $10(45.45 \%)[N=22]$ & $10(45.45 \%)[N=22]$ \\
\hline Disease duration, months (Median, IQR) & $11.3(3.7,23.7)$ & $9.5(2.9,20.3)$ & $12.0(3.9,36.2)$ \\
\hline Active peripheral joint count (Median, IQR) & $0.0(0.0,2.0)$ & $0.0(0.0,2.0)$ & $0.0(0.0,2.0)$ \\
\hline Tender entheses (Median, IQR) & $1.5(0.0,4.0)$ & $0.0(0.0,5.0)$ & $2.0(0.0,4.0)$ \\
\hline Physician global (0-10; Median, IQR) & $2.0(2.0,4.0)[N=34]$ & $2.0(2.0,4.0)[N=21]$ & $2.0(1.0,3.0)[N=13]$ \\
\hline Patient global (0-10; Median, IQR) & $4.3(2.0,6.0)[N=30]$ & $5.0(3.0,6.0)[N=19]$ & $2.0(1.0,7.0)[N=11]$ \\
\hline Patient pain (0-10; Median, IQR) & $5.0(1.6,7.0)[N=30]$ & $5.6(2.0,7.0)[N=19]$ & $3.0(1.0,7.0)[N=11]$ \\
\hline csDMARD use during follow-up & $17(36.96 \%)$ & $8(34.78 \%)$ & $9(39.13 \%)$ \\
\hline Non-TNFi bDMARD use during follow-up* & $4(8.70 \%)$ & $4(17.39 \%)$ & $0(0.00 \%)$ \\
\hline NSAID use during follow-up & $32(69.57 \%)$ & $12(52.17 \%)$ & $20(86.96 \%)$ \\
\hline
\end{tabular}

Legend. *There were four patients exposed to a non-TNFi bDMARD; all were also TNFi-exposed. Three of four started the TNFi first and of those, two did not start the non-TNFi until after the second MRI that was used for the change in inflammation assessment 
Table 2 Unadjusted inflammation change scores

\begin{tabular}{|c|c|c|c|c|}
\hline & All subjects & TNFi exposed & TNFi unexposed & \\
\hline & Median SIS Change Score (IQR) & & & $p$-value \\
\hline All subjects & $0.0(-14.7,0.0)[N=45]$ & $-5.2(-24.7,0.0)[N=22]$ & $0.0(-10.3,4.0)[N=23]$ & 0.09 \\
\hline Baseline $S I S \geq 2^{*}$ & $-16.8(-26.0,-10.3)[N=22]$ & $-20.7(-26.0,-11.0)[N=13]$ & $-14.3(-20.2,-9.3)[N=9]$ & 0.09 \\
\hline \multirow[t]{2}{*}{ Baseline SIS =0 } & $0.0(0.0,4.0)[N=23]$ & $0.0(0.0,0.0)[N=9]$ & $0.0(0.0,4.0)[N=14]$ & 0.12 \\
\hline & Frequency meeting MCID (\%) & & & $p$-value \\
\hline All subjects & $19(42.22 \%)[N=45]$ & $11(50.00 \%)[N=22]$ & $8(34.78 \%)[N=23]$ & 0.30 \\
\hline Baseline SIS>0 & $19(86.36 \%)[N=22]$ & $11(84.62 \%)[N=13]$ & 8 (88.89\%) [N=9] & 0.77 \\
\hline
\end{tabular}

Legend. MRI sequence availability dictated which subjects were evaluated with SIS and SSS detailed scoring. Of the 46 unique subjects, one was missing the necessary sequences to perform SIS detailed scoring. *SPARCC SIS score $\geq 2$ is used as a surrogate for MRI-sacroiliitis positive in clinical trials [23]

those with SIS $<2$ on baseline imaging did not present with a statistically significant difference in clinical disease activity as measured by physician global disease activity assessment (median $=3$ [IQR: 2-4] vs 2 [IQR: $2-3$ ], $p=0.33$ ). The median SIS at baseline in all patients was 0 (IQR: 0-19) and in those with SIS $\geq 2$ was 19.9 (13.3-28.5), respectively. Nineteen (42.2\%), 10 (22.2\%), and $16(35.6 \%)$ cases demonstrated improvement, worsening, or no change in the SIS over time, respectively. Subjects whose SIS improved over time had a median decrease in SIS of 20.2 (IQR: 11-27). Subjects whose SIS worsened had a median increase in SIS of 8 (IQR: 4-14.5). Of those with worsening of the SIS from 0 to $\geq 1(N=7)$, one was exposed to a TNFi but was off therapy for nine months preceding the second MRI. Correlation of change in patient-reported pain with change in SIS was weak $(r=0.14)$.

Unadjusted inflammation change scores are presented in Table 2. The median change in SIS in TNFi exposed and unexposed subjects with a baseline SIS $\geq 0$ was 20.7 and -14.3 , respectively $(p=0.09)$. Eleven $(85 \%)$ TNFi-exposed and 8 (89\%) TNFi-unexposed subjects with a baseline SIS $\geq 0$ met the SIS MCID of change $\geq-$ 2.5. Of the patients who met the MCID, 81.8 and $50 \%$ of TNFi exposed and unexposed, respectively, had resolution of inflammation. Table 3 shows clinical details of the TNFi unexposed patients who met the SIS MCID. One unexposed subject received TNFi for 67 days but did not meet the protocol-defined definition of TNFi exposure ( 290 days).

Using the ATE model adjusted for baseline SIS, the average effect of TNFi on SIS in all patients was -7.85 (95\% CI: $-12.22,-3.49 ; p<0.01)$. The ATE for patients with a baseline SIS $\geq 2$ was -14.50 (95\% CI: -21.62 , -7.37 ; $p<0.01)$ and for those with a baseline SIS $<2$ was -1.27 (95\% CI: $-4.37,1.83 ; p=0.42$ ) (Table 4). Sensitivity analyses with definitions of TNFi exposure as $\geq 60, \geq 120$ and $\geq$ 180 days demonstrated nearly identical results with baseline SIS $\geq 2$ with ATE of -15.22 (95\% CI:-22.10, -8.33 ; $p<0.001),-15.99$ (95\% CI: $-23.04,-8.95 ; p<0.001)$, and -14.47 (95\% CI: $-18.78,-10.16 ; p<0.001$ ), respectively. Sensitivity analysis of patients excluding the 2 patients in the TNFi exposed group who were also exposed to a nonTNFi biologic between MRI studies demonstrated similar results with ATE of $-8.09,-14.5$, and -1.10 for all patients, patients with baseline SIS $\geq 2$ and baseline SIS $<2$, respectively.

A spaghetti plot of SIS stratified by TNFi exposure suggests a consistency with these results, demonstrating a negative slope of fitted line in TNFi exposed patients and a positive slope in TNFi unexposed patients (Fig. 1).

Table 3 Subjects without TNFi exposure who met SIS MCID

\begin{tabular}{|c|c|c|c|c|c|c|c|c|c|c|}
\hline Subject & Sex & $\begin{array}{l}\text { Time between MRI scans } \\
\text { (months) }\end{array}$ & $\begin{array}{l}\text { Pain change } \\
\text { score }\end{array}$ & $\begin{array}{l}\text { Baseline } \\
\text { SIS }\end{array}$ & $\begin{array}{l}\text { Follow- } \\
\text { up SIS }\end{array}$ & $\begin{array}{l}\text { Baseline } \\
\text { Erosion }\end{array}$ & $\begin{array}{l}\text { Follow-up } \\
\text { Erosion }\end{array}$ & $\begin{array}{l}\text { NSAI } \\
\text { Ds }\end{array}$ & CDMARD & $\begin{array}{l}\text { TNFi exposure } \\
\text { (days) }\end{array}$ \\
\hline 1 & $M$ & 8.5 & - & 34.7 & 7 & 11 & 4.3 & None & None & 0 \\
\hline 2 & $M$ & 33.8 & -4 & 14.3 & 0 & 5.3 & 5.7 & Yes & MTX & 0 \\
\hline 3 & M & 22.1 & - & 3 & 0 & 0 & 0 & Yes & SSZ & 0 \\
\hline 4 & $M$ & 5.8 & - & 13.3 & 4 & 5 & 9 & Yes & None & 0 \\
\hline 5 & M & 36.0 & - & 30.7 & 16 & 0 & 0 & Yes & None & 0 \\
\hline 6 & $\mathrm{~F}$ & 35.7 & - & 24.7 & 4.5 & 0 & 4.7 & Yes & SSZ & 0 \\
\hline 7 & M & 7.5 & -1 & 10.3 & 0 & 0 & 0 & Yes & None & 0 \\
\hline 8 & $\mathrm{~F}$ & 2.9 & -1 & 28.5 & 0 & 10 & 13 & Yes & MTX & 67 \\
\hline
\end{tabular}


Table 4 Treatment effects

\begin{tabular}{llll}
\hline & $\mathbf{N}$ & Average treatment effect $(\mathbf{9 5} \% \mathrm{Cl})$ & $\mathbf{p}$-value \\
\hline SIS & & & \\
All & 42 & $-7.85(-12.22,-3.49)$ & 0.00 \\
Baseline SIS $\geq 2 *$ & 21 & $-14.50(-21.62,-7.37)$ & 0.00 \\
Baseline SIS $<2$ & 21 & $-1.27(-4.37,1.83)$ & 0.42 \\
SSS & & & \\
$\quad$ Erosion & 18 & $0.72(-1.88,3.31)$ & 0.588 \\
Sclerosis & 18 & $-0.18(-0.93,0.56)$ & 0.628
\end{tabular}

Legend. Average treatment effects from TNFi use, adjusting for baseline SIS or SSS lesions scores as appropriate. Exposure defined as $\geq 90$ days of TNFi use. *SPARCC SIS score $\geq 2$ is used as a surrogate for MRI-sacroiliitis positive in clinical trials [23]

\section{SPARCC structural scores}

Eighteen subjects, 14 TNFi exposed and 4 TNFi unexposed, with two MRI studies at least 2 years apart were included in the analysis of SSS lesions. Four (28.6\%) TNFi exposed and $0(0.0 \%)$ TNFi unexposed patients had erosion scores $>0$ at baseline. Of the four patients with a baseline erosion score $>0,2$ (50.0\%) demonstrated improvement and $2(50.0 \%)$ demonstrated worsening in the erosion score over time. In cases where the baseline erosion score was zero, all 4 (100.0\%) of the unexposed patients and $2(20.0 \%)$ of the TNFi exposed patients had an erosion score $>0$ on follow-up imaging. Unadjusted median SSS erosion change scores in TNFi unexposed subjects signaled a statistically significant $(p=0.03)$ overall worsening in erosion score (5.3 IQR: 4.0, 7.5) whereas the TNFi exposed subjects experienced very little change in erosion score $(0.0 \mathrm{IQR}, 0.0,2.6)$. As mentioned previously, all four (100.0\%) of the TNFi unexposed patients worsened. In the TNFi exposed group, $4(28.6 \%)$ worsened, $8(57.1 \%)$ stayed the same, and $2(14.3 \%)$ improved. Spaghetti plots of SSS erosion trajectory stratified by TNFi exposure suggest a consistency with these results, demonstrating a positive slope (worsening) in TNFi-unexposed subjects and a relatively flat slope (no change) in TNFi exposed subjects for the respective fitted lines (Fig. 2). Using the ATE model adjusted for baseline erosion score, the average effect of TNFi on SSS erosion was not significant and was 0.72 (95\% CI, $-1.88,3.31 ; p=0.59$; Table 4). Sensitivity analysis of patients excluding the 3 patients in the TNFi exposed group who were also exposed to a non-TNFi biologic between MRI studies demonstrated similar results with ATE of 1.20 (95\% CI, - 1.60, 4.00; $p=0.40)$.

Three (21.4\%) TNFi exposed patients and $0(0.0 \%)$ TNFi unexposed patients had sclerosis scores $>0$ at baseline. Of these three cases where the baseline sclerosis score was >0, 2 (66.7\%) demonstrated improvement and 1 (33.3\%) demonstrated worsening in the sclerosis score over time. In cases where the baseline sclerosis score was zero, none $(0.0 \%)$ of the TNFi unexposed patients and one of the TNFi exposed patients

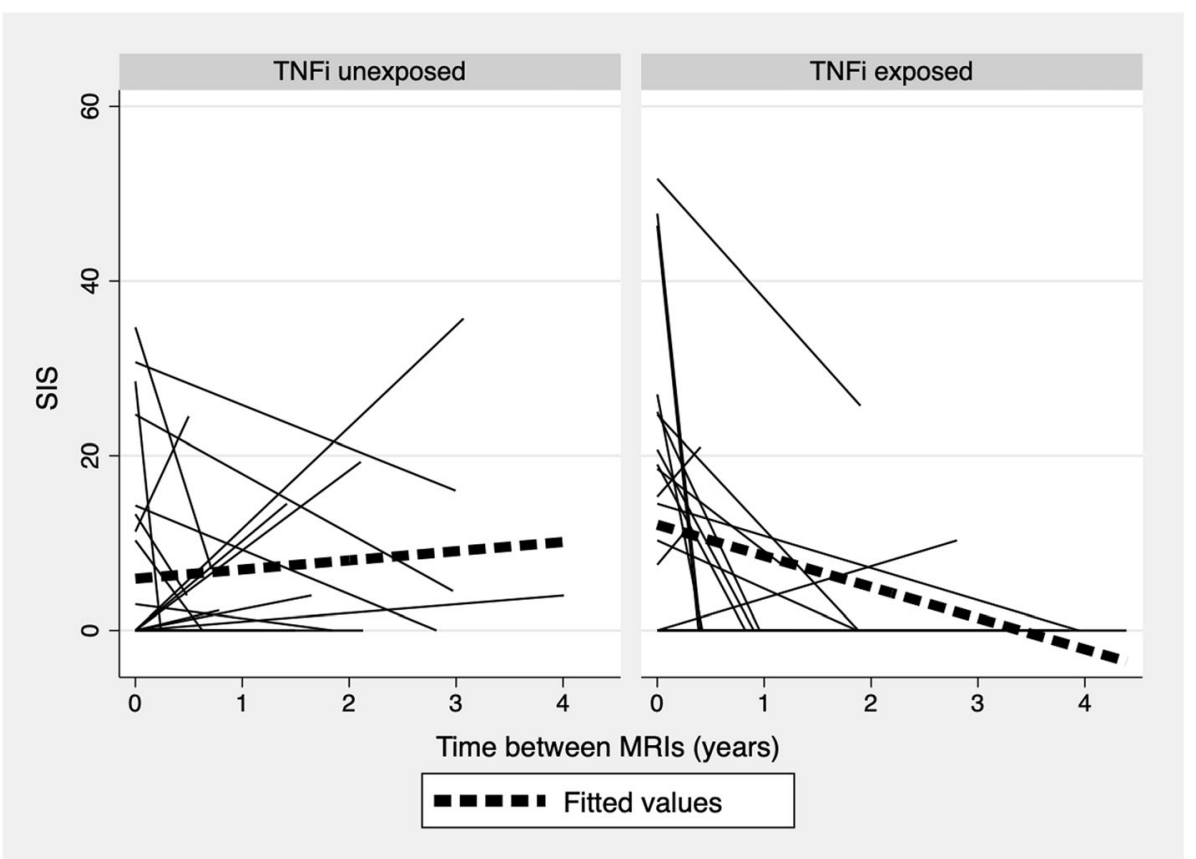

Fig. 1 Unadjusted SIS trajectory of all subjects $(N=45)$. Each solid line begins at a subject's baseline SIS score and ends at their follow-up score. Dotted lines are the overall fitted regression lines. The TNFi exposure was defined as $\geq 90$ days of use between MRIs. SIS = sacroiliac joint inflammation score; TNFi = tumor necrosis factor inhibitor 


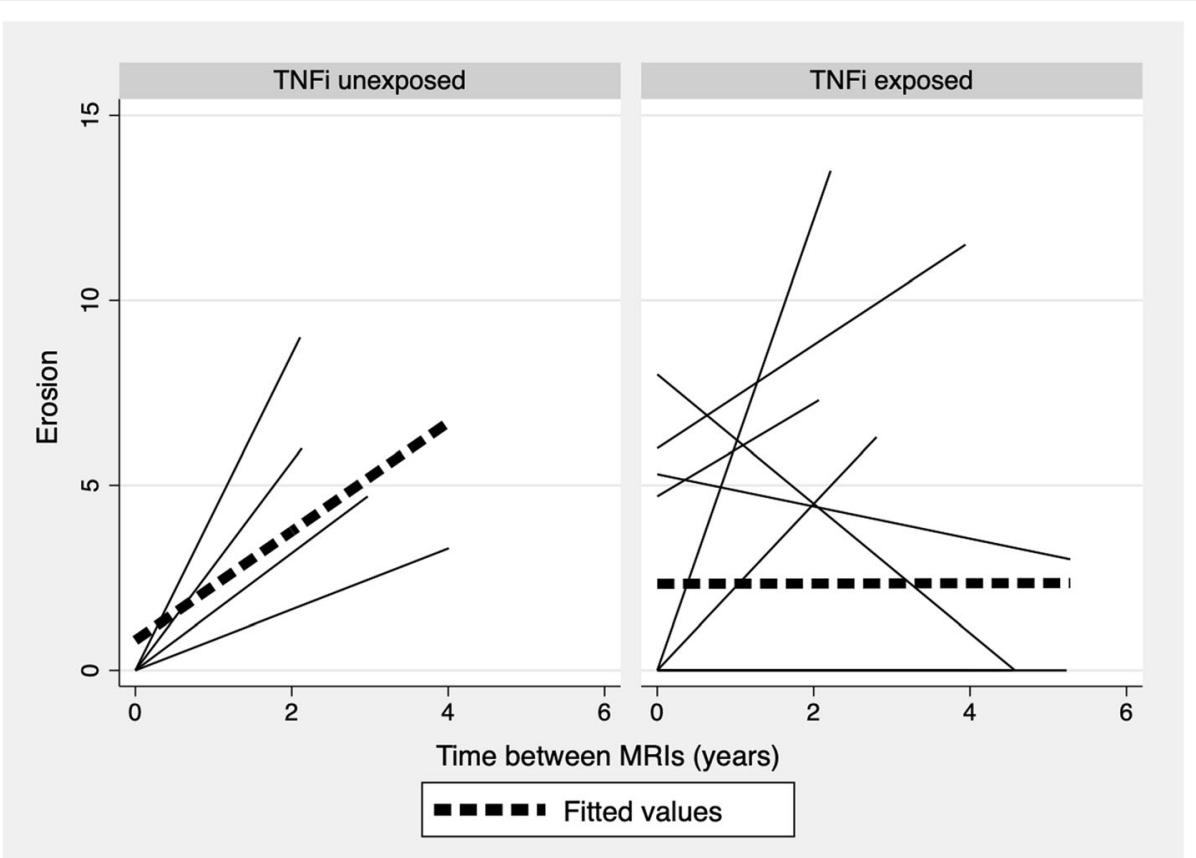

Fig. 2 Unadjusted trajectory of SSS erosion in all subjects $(N=38)$. Each solid line begins at a subject's baseline SSS erosion score and ends at their follow-up score. Dotted lines are the overall fitted regression lines. The TNFi exposure was defined as $\geq 90$ days of use between MRIs. SSS = sacroiliac joint inflammation score; TNFi = tumor necrosis factor inhibitor

had a sclerosis score $>0$ at the time of follow-up imaging. The median unadjusted SSS sclerosis change scores in TNFi exposed and unexposed subjects were both 0.0 (95\% CI: 0.0, 0.0). All four (100.0\%) of the TNFi unexposed patients had unchanged zero sclerosis scores during follow-up. In the TNFi exposed group, 2 (14.3\%) worsened, $10(71.4 \%)$ stayed the same, and 2 (14.3\%) improved. Using the ATE model adjusted for baseline sclerosis score, the average effect of TNFi on SSS sclerosis was not significant and was - 0.18 (95\% CI:-0.93, 0.56; $p=0.63$; Table 4). Sensitivity analysis of patients excluding the 3 patients in the TNFi exposed group who were also exposed to a non-TNFi biologic between MRI studies demonstrated similar results with ATE of -0.09 (95\% CI: $-0.92,0.74 ; p=$ 0.82).

Models were not run on ankylosis, backfill, or fat metaplasia due to the small number of lesions in the cohort.

\section{Discussion}

Our objective was to describe MRI changes over time in inflammatory and structural lesions at the sacroiliac joints in children with SpA exposed and unexposed to TNFi. Data on longitudinal changes at the pediatric SIJ are sparse and greatly needed. In our small, uncontrolled cohort, we observed that differences exist in SIJ lesions between TNFi-exposed and TNFi-unexposed patients. Raw, median inflammation change scores showed more improvement in TNFi-exposed patients that approached statistical significance and TNFi use was also associated with a significant average treatment effect in inflammation on MRI at the sacroiliac joints in juvenile SpA. Unadjusted median erosion change scores were significantly higher in TNFi unexposed versus exposed subjects but in the ATE model adjusted for baseline erosion score the effect of TNFi was not significant. Although ATE model is most appropriate for randomized trials, here we applied this model only for purpose of exploration rather than making formal hypothesis testing. Stabilization of the erosion score without progression is also a positive clinical outcome. These results support the use of change in SIS and SSS as objective tools to assess response to biologics in effectiveness and efficacy studies.

Lesion detection reliability across raters was in line with expected thresholds for lesions that were not rarely reported in this cohort. Sclerosis and backfill had the lowest levels of agreement which can most likely be attributed to the low prevalence reported by any rater. The remaining SPARCC SIS and SSS components met the literature reported agreement of fair to excellent [20-22].

Surprisingly, the majority of TNFi unexposed subjects with a baseline SIS $\geq 0$ met the SIS minimal clinically important difference (MCID $\geq 2.5$ ), and this will need to be explored in future studies. Half (4 of 8 ) of unexposed 
patients achieving the MCID demonstrated total resolution of inflammation on the follow-up study. One of these subjects had mild inflammation at baseline (SIS = 3 ) and one was actually TNFi exposed but did not meet the study's definition of exposure ( $\geq 90$ days). The results in the unexposed group underscore the importance of ascertaining the natural history of inflammatory change in the pediatric SIJ as this is currently unknown. Based on clinical experience and studies in adults, TNFis provide symptomatic relief to patients with sacroiliitis, but perhaps not all patients require biologic therapy for resolution to occur. Patients in this study were often treated with an NSAID if they weren't also on a biologic. There is limited evidence from studies in adults with ankylosing spondylitis that NSAIDs, particularly celecoxib, may play a role in slowing progression of structural damage $[25,26]$ though data from a more recent study did not confirm these findings [27]. The role of monotherapy with NSAIDs has not been evaluated in children with sacroiliitis and is unlikely to be studied since the most recent ACR guidelines strongly recommend initiation of a biologic over NSAID monotherapy [28]. Future well-designed studies need to ascertain which patients are most likely to have persistent inflammation and would benefit from biologic initiation.

Validated measures to assess progression of sacroiliitis include the New York (NY) criteria [19], the SPARCC sacroiliac joint inflammation score [19], and the SPARCC sacroiliac joint structural score. The NY criteria are used on radiographs, only assess structural damage, and have moderate interrater reliability [29]. Despite its limitations, the NY criteria remain the gold standard for classification of ankylosing spondylitis and assessment of radiographic progression in clinical trials [30]. There are preliminary data signaling that use of a biologic compared to non-biologic agent may slow radiographic progression, but the effect is only seen when the gap between radiographs is quite long (approximately 4 years) and serial radiographs cannot inform about change in active inflammation [31]. The SPARCC SIS is being increasingly used to assess response at the sacroiliac joints in clinical trials and offers an objective measure of change that can be assessed over a much shorter time period than radiographs. Several studies have demonstrated the ability to detect significant changes in the SPARCC SIS in as little as 12 and 16 weeks after initiation of a biologic in adults with nonradiographic SpA [12, 32-34]. Our results support the use of the SIS in the pediatric population with axial arthritis as well. To date, there have been no pediatric randomized clinical trials of biologic agents for axial arthritis that include evaluation of serial imaging. Objective imaging measures of response to these novel agents are critical to assess since complaints of inflammatory back pain by history and pain to palpation have relatively low positive predictive value in the pediatric population [10]. Use of these subjective measures instead of objective measures like the SPARCC SIS may increase the likelihood of missing significant findings.

One limitation of this study was the rarity of structural lesions other than erosion. As such, we were unable to run our models to assess for the association of cumulative TNFi exposure with change over time in most of these lesions. Our inability to show significant association of TNFi with change in erosions and sclerosis scores using the adjusted ATE model may be because we were underpowered to detect the association or perhaps, as suggested by prior work [15], TNFis do not halt structural progression. However, it is notable that in Fig. 2 , the positive slope of the fitted line indicates worsening for erosion in TNFi unexposed cases, while the flat slope of the fitted line in the TNFi exposed patients indicates that the progression, on average, is at least slowed. A larger sample size and a cohort with a greater prevalence of structural damage and longer disease duration and/or follow-up is needed to further evaluate the associations of cumulative TNFi exposure and change over time in these lesions.

The retrospective observational study design raises a few additional limitations that should be considered. First, there was variability in the imaging protocols performed at each of the three institutions and over time within institutions. The ability to score the SPARCC SIS and SSS is primarily dependent on the presence of coronal oblique T1W and fluid-sensitive sequences of the sacroiliac joints; since these sequences were present in all studies per our inclusion criteria, the validity of our results are not likely to be impacted by minor imaging protocol differences. All studies were performed on high field-strength magnets $(1.5 \mathrm{~T}$ or $3.0 \mathrm{~T})$. Second, there is the potential for confounding by indication whereby patients in the TNFi-exposed group had more severe disease. However, there were no statistically significant differences in clinical features between groups and, if residual unmeasured confounders were present, this would bias the study toward the null hypothesis of no difference in change in SIS or SSS. Third, the time between imaging studies and duration and magnitude of TNFi exposure varied as the studies were ordered as per clinical care. There is likely also selection bias introduced into the cohort based on which patients are more likely to have a repeat MRI and receive more aggressive treatment. In the collective authors' experience, insurance will often not pay for an MRI to confirm resolution of inflammation or in the absence of symptoms, so most repeat MRIs are performed in patients who are having symptoms. That practice, however, would bias the 
results towards the null or finding no difference in those exposed and unexposed to TNFi therapy. Another limitation that could result in a diminished observed effect in our analysis is that it was difficult to account for treatment regimen non-compliance. Some physician notes detailed incidents of missed doses for a variety of reasons and other patients may have intentionally not been taking their medication as prescribed. The effect on the sacroiliac joints of these gaps in therapy or nonadherence to treatment plan is unknown. Lastly, several subjects in the TNFi exposed group received more than one TNFi sequentially between eligible MRI studies, most likely indicating poor response to therapy again biasing our results towards the null and making the ability to detect a difference between the two groups even more impressive.

\section{Conclusion}

In summary, we describe how MRI lesions in the SIJ change under different treatment conditions. TNFi use was associated with a significant average treatment effect in inflammation at the sacroiliac joints in juvenile SpA. We also observed a trend, albeit insignificant, in TNFi exposure and a halted progression in the SSS erosion score for studies done at least two years apart. Incorporation of the SIS and SSS into not only clinical trials but also effectiveness studies should be strongly considered as they provide feasible and responsive means of objectively assessing response over a short period of time. An enhanced understanding of the magnitude and rate of response to TNFi in children will help to inform the design of clinical trials.

\section{Abbreviations \\ SpA: spondyloarthritis; TNFi: tumor necrosis factor inhibitor; MRI: magnetic resonance imaging; ATE: average treatment effects; \\ SPARCC: Spondyloarthritis Research Consortium of Canada; SSS: sacroiliac joint inflammation score SIS and structural score; DICOM: Digital Imaging and Communication in Medicine; STIR: Short-tau inversion recovery; BME: bone marrow edema; ICCs: Intraclass correlation coefficients; IQR: Interquartile range; Cl: Confidence interval}

\section{Acknowledgements \\ The authors would like to acknowledge Joel Paschke for his assistance with organizing and managing the digital imaging files and scoring data within the CaRE Arthritis platform, Joshua Pavlovec (Children's of Alabama) for his help in identifying and obtaining eligible MRI files, and Lester Liao (University of Alberta) for abstracting and transferring subject data from his site.}

\section{Authors' contributions}

TGB helped with the analysis and interpretation of data and drafting of the manuscript. RX helped with design of the work, analysis, and interpretation of the data. RGP helped with the analysis and interpretation of the data and drafting of the manuscript. NC, MF and DB helped with acquisition and interpretation of the data. DGR and MLS helped with acquisition of the data. PFW helped with the design of the work, analysis, and interpretation of the data, drafting of the manuscript. All authors have approved the submitted version of the manuscript.
Funding

This study was funded by the Rheumatology Research Foundation (P.F. Weiss).

\section{Availability of data and materials}

The data that support the findings of this study are available from the corresponding author but restrictions apply to the availability of these data, which were used under data use agreements for the current study, and so are not publicly available. Data are however available from the authors upon reasonable request and with permission of all sites contributing data.

\section{Declarations}

\section{Ethics approval and consent to participate}

This study's protocol was reviewed and approved by the University of Alabama at Birmingham's (IRB-160701011) and Children's Hospital of Philadelphia's (IRB 16-013015) Committees for the Protection of Human Subjects and the ethics committee at the University of Alberta (REB Pro00081651). Waivers of consent and HIPAA authorization were granted as procedures represented minimal risk to the subjects and did not adversely affect the rights and welfare of the subjects.

\section{Consent for publication}

Not applicable.

\section{Competing interests}

Dr. Weiss has served as a consultant for Lilly and Novartis.

\section{Author details}

${ }^{1}$ Division of Rheumatology and Center for Pediatric Clinical Effectiveness at the Children's Hospital of Philadelphia, Department of Pediatrics, Philadelphia, USA. ²Department of Biostatistics, Epidemiology and Informatics, Perelman School of Medicine, University of Pennsylvania, Philadelphia, USA. ${ }^{3}$ Division of Rheumatology at the Children's Hospital of Philadelphia, Department of Pediatrics, Philadelphia, USA. ${ }^{4}$ Department of Radiology at Penn State Health Milton S. Hershey Children's Hospital, Hershey, PA, USA. ${ }^{5}$ Department of Radiology at the Children's Hospital of Philadelphia and Department of Radiology, Perelman School of Medicine at the University of Pennsylvania, Philadelphia, USA. ${ }^{6}$ Division of Pediatric Rheumatology, Department of Pediatrics, University of Alberta, Edmonton, Alberta, Canada. ${ }^{7}$ Division of Pediatric Rheumatology, Department of Pediatrics, University of Alabama at Birmingham, Birmingham, AL, USA. ${ }^{8}$ Department of Pediatrics, Division of Rheumatology and Center for Pediatric Clinical Effectiveness at the Children's Hospital of Philadelphia and Center for Clinical Epidemiology and Biostatistics, Perelman School of Medicine at the University of Pennsylvania, 2716 South Street, Room 11121, Philadelphia, PA 19104, USA.

Received: 27 September 2021 Accepted: 9 November 2021

Published online: 02 December 2021

\section{References}

1. Pagnini I, Savelli S, Matucci-Cerinic M, Fonda C, Cimaz R, Simonini G. Early predictors of juvenile sacroiliitis in enthesitis-related arthritis. J Rheumatol. 2010;37(11):2395-401. https://doi.org/10.3899/jrheum.100090.

2. Flato B, Hoffmann-Vold AM, Reiff A, Forre O, Lien G, Vinje O. Long-term outcome and prognostic factors in enthesitis-related arthritis: a case-control study. Arthritis Rheum. 2006;54(11):3573-82. https://doi.org/10.1002/art.22181.

3. Stoll ML, Bhore R, Dempsey-Robertson M, Punaro M. Spondyloarthritis in a pediatric population: risk factors for sacroiliitis. J Rheumatol. 2010;37(11): 2402-8. https://doi.org/10.3899/jrheum.100014.

4. Bollow M, Braun J, Biedermann T, Mutze S, Paris S, Schauer-Petrowskaja C, et al. Use of contrast-enhanced MR imaging to detect sacroiliitis in children. Skelet Radiol. 1998;27(11):606-16. https://doi.org/10.1007/s002560050446.

5. Stone M, Warren RW, Bruckel J, Cooper D, Cortinovis D, Inman RD. Juvenileonset ankylosing spondylitis is associated with worse functional outcomes than adult-onset ankylosing spondylitis. Arthritis Rheum. 2005;53(3):445-51. https://doi.org/10.1002/art.21174.

6. Chen HA, Chen CH, Liao HT, Lin YJ, Chen PC, Chen WS, et al. Clinical, functional, and radiographic differences among juvenile-onset, adult-onset, 
and late-onset ankylosing spondylitis. J Rheumatol. 2012;39(5):1013-8. https://doi.org/10.3899/jrheum.111031.

7. Jadon DR, Shaddick G, Jobling A, Ramanan AV, Sengupta R. Clinical outcomes and progression to orthopedic surgery in juvenile- versus adultonset ankylosing spondylitis. Arthritis Care Res (Hoboken). 2015;67(5):651-7. https://doi.org/10.1002/acr.22500.

8. Lin YC, Liang TH, Chen WS, Lin HY. Differences between juvenile-onset ankylosing spondylitis and adult-onset ankylosing spondylitis. Journal of the Chinese Medical Association : JCMA. 2009;72(11):573-80. https://doi.org/10.1 016/S1726-4901(09)70432-0.

9. Gensler LS, Ward MM, Reveille JD, Learch TJ, Weisman MH, Davis JC Jr. Clinical, radiographic and functional differences between juvenile-onset and adult-onset ankylosing spondylitis: results from the PSOAS cohort. Ann Rheum Dis. 2008;67(2):233-7. https://doi.org/10.1136/ard.2007.072512.

10. Weiss PF, Xiao R, Biko DM, Chauvin NA. Assessment of Sacroiliitis at diagnosis of juvenile Spondyloarthritis by radiography, magnetic resonance imaging, and clinical examination. Arthritis Care Res (Hoboken). 2016;68(2): 187-94. https://doi.org/10.1002/acr.22665.

11. O'Shea FD, Boyle E, Riarh R, Tse SM, Laxer RM, Inman RD. Comparison of clinical and radiographic severity of juvenile-onset versus adult-onset ankylosing spondylitis. Ann Rheum Dis. 2009:68(9):1407-12. https://doi.org/1 0.1136/ard.2008.092304.

12. Sieper J, van der Heijde D, Dougados M, Maksymowych WP, Scott BB, Boice $J A$, et al. A randomized, double-blind, placebo-controlled, sixteen-week study of subcutaneous Golimumab in patients with active nonradiographic axial Spondyloarthritis. Arthritis Rheumatol. 2015;67(10):2702-12. https://doi. org/10.1002/art.39257.

13. Pedersen SJ, Poddubnyy D, Sørensen IJ, Loft A-GG, Hindrup JS, Thamsborg $G$, et al. Course of Magnetic Resonance Imaging-Detected Inflammation and Structural Lesions in the Sacroiliac Joints of Patients in the Randomized, Double-Blind, Placebo-Controlled Danish Multicenter Study of Adalimumab in Spondyloarthritis, as Assessed by the Berlin and Spondyloarthritis Research Consortium of Canada Methods. Arthritis \& rheumatology (Hoboken, NJ). 2016:68(2):418-29.

14. Maksymowych WP, Dougados M, van der Heijde D, Sieper J, Braun J, Citera $\mathrm{G}$, et al. Clinical and MRI responses to etanercept in early non-radiographic axial spondyloarthritis: 48-week results from the EMBARK study. Ann Rheum Dis. 2016;75(7):1328-35. https://doi.org/10.1136/annrheumdis-2015-207596.

15. Bray TJP, Lopes A, Fisher C, Ciurtin C, Sen D, Hall-Craggs MA. Sacroiliac joint Ankylosis in young Spondyloarthritis patients receiving biologic therapy: observation of serial magnetic resonance imaging scans. Arthritis Rheumatol. 2019;71(4):594-8. https://doi.org/10.1002/art.40750.

16. Weiss PF, Xiao R, Brandon TG, Biko DM, Maksymowych WP, Lambert RG, et al. Radiographs in screening for sacroiliitis in children: what is the value? Arthritis research \& therapy. 2018;20(1):141. https://doi.org/10.1186/s13075018-1642-8.

17. Maksymowych WP, Lambert RG, Brown LS, Pangan AL. Defining the minimally important change for the SpondyloArthritis research consortium of Canada spine and sacroiliac joint magnetic resonance imaging indices for ankylosing spondylitis. J Rheumatol. 2012;39(8):1666-74. https://doi.org/1 0.3899/jheum.120131.

18. Maksymowych WP, Wichuk S, Dougados M, Jones HE, Pedersen R, Szumsk $A$, et al. Modification of structural lesions on MRI of the sacroiliac joints by etanercept in the EMBARK trial: a 12-week randomised placebo-controlled trial in patients with non-radiographic axial spondyloarthritis. Ann Rheum Dis. 2017;77(1):78-84. https://doi.org/10.1136/annrheumdis-2017-211605.

19. Maksymowych WP, Wichuk S, Chiowchanwisawakit P, Lambert RG, Pedersen SJ. Development and preliminary validation of the spondyloarthritis research consortium of Canada magnetic resonance imaging sacroiliac joint structural score. J Rheumatol. 2015:42(1):79-86. https://doi.org/10.3899/ jrheum. 140519.

20. Weiss PF, Maksymowych WP, Lambert RG, Jaremko JL, Biko DM, Paschke J, et al. Feasibility and reliability of the Spondyloarthritis research consortium of Canada sacroiliac joint structural score in children. J Rheumatol. 2018; 45(10):1411-7. https://doi.org/10.3899/jheum.171329.

21. Weiss PF, Maksymowych WP, Lambert RG, Jaremko JL, Biko DM, Paschke J, et al. Feasibility and reliability of the Spondyloarthritis research consortium of Canada sacroiliac joint inflammation score in children. Arthritis Research \& Therapy. 2018;20(1):56. https://doi.org/10.1186/s13075-018-1543-x.

22. Weiss PF, Maksymowych WP, Xiao R, Biko DM, Francavilla ML, Lambert RG, et al. Spondyloarthritis research consortium of Canada sacroiliac joint inflammation and structural scores: change score reliability and recalibration utility in children. Arthritis Res Ther. 2020;22(1):58. https://doi.org/10.1186/ s13075-020-02157-4.

23. van den Berg R, de Hooge M, Bakker PA, van Gaalen F, Navarro-Compan V, Fagerli KM, et al. Metric properties of the SPARCC score of the sacroiliac joints - data from baseline, 3-month, and 12-month Followup in the SPACE cohort. J Rheumatol. 2015;42(7):1186-93. https://doi.org/10.3899/jrheum.14 0806.

24. B R. Fundamentals of Biostatistics. Belmont, CA: Tomson-Brooks/Cole; 2006.

25. Kroon F, Landewé R, Dougados M, van der Heijde D. Continuous NSAID use reverts the effects of inflammation on radiographic progression in patients with ankylosing spondylitis. Ann Rheum Dis. 2012;71(10):1623-9. https:/doi. org/10.1136/annrheumdis-2012-201370.

26. Wanders A, Heijde D, Landewé R, Béhier JM, Calin A, Olivieri I, et al. Nonsteroidal antiinflammatory drugs reduce radiographic progression in patients with ankylosing spondylitis: a randomized clinical trial. Arthritis Rheum. 2005;52(6):1756-65. https://doi.org/10.1002/art.21054.

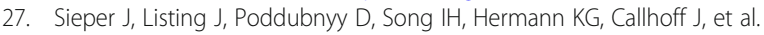
Effect of continuous versus on-demand treatment of ankylosing spondylitis with diclofenac over 2 years on radiographic progression of the spine: results from a randomised multicentre trial (ENRADAS). Ann Rheum Dis. 2016;75(8):1438-43. https://doi.org/10.1136/annrheumdis-2015-207897.

28. Ringold S, Angeles-Han ST, Beukelman T, Lovell D, Cuello CA, Becker ML, et al. 2019 American College of Rheumatology/Arthritis Foundation guideline for the treatment of juvenile idiopathic arthritis: therapeutic approaches for non-systemic polyarthritis, Sacroiliitis, and Enthesitis. Arthritis Rheumatol. 2019;71(6):846-63. https://doi.org/10.1002/art.40884.

29. van den Berg R, Lenczner G, Feydy A, van der Heijde D, Reijnierse M, Saraux A, et al. Agreement between clinical practice and trained central reading in reading of sacroiliac joints on plain pelvic radiographs. Results from the DESIR cohort. Arthritis Rheumatol. 2014;66(9):2403-11. https://doi.org/10.1 002/art.38738.

30. Poddubnyy D, Sieper J. Radiographic progression in ankylosing spondylitis/ axial spondyloarthritis: how fast and how clinically meaningful? Curr Opin Rheumatol. 2012;24(4):363-9. https://doi.org/10.1097/BOR.0b013e328352b7bd.

31. Haroon N, Inman RD, Learch TJ, Weisman MH, Lee M, Rahbar MH, et al. The impact of tumor necrosis factor alpha inhibitors on radiographic progression in ankylosing spondylitis. Arthritis Rheum. 2013;65(10):2645-54. https://doi.org/10.1002/art.38070.

32. Pedersen SJ, Poddubnyy D, Sorensen IJ, Loft AG, Hindrup JS, Thamsborg G, et al. Course of MRI inflammation and structural lesions in the sacroiliac joints in a randomized double-blind placebo-controlled trial of adalimumab in patients with axial Spondyloarthritis as assessed by the Berlin and SPARCC methods (the DANISH study). Arthritis Rheumatol. 2015;68(2):41829. https://doi.org/10.1002/art.39434.

33. Lambert RG, Salonen D, Rahman P, Inman RD, Wong RL, Einstein SG, et al. Adalimumab significantly reduces both spinal and sacroiliac joint inflammation in patients with ankylosing spondylitis: a multicenter, randomized, double-blind, placebo-controlled study. Arthritis Rheum. 2007; 56(12):4005-14. https://doi.org/10.1002/art.23044.

34. van der Heijde D, Deodhar A, Wei JC, Drescher E, Fleishaker D, Hendrikx T, et al. Tofacitinib in patients with ankylosing spondylitis: a phase II, 16-week, randomised, placebo-controlled, dose-ranging study. Ann Rheum Dis. 2017; 76(8):1340-7. https://doi.org/10.1136/annrheumdis-2016-210322.

\section{Publisher's Note}

Springer Nature remains neutral with regard to jurisdictional claims in published maps and institutional affiliations. 